\title{
Protect to detect: A Golgi apparatus targeted probe to image mobile zinc through the use of a lipophilic cell-labile protecting group strategy
}

\author{
Le Fang, ${ }^{a}$ Rachel Crespo-Otero, ${ }^{a}$ Christopher R Jones ${ }^{a}$ and Michael Watkinson*b \\ a The Joseph Priestley Building, School of Biological and Chemical Science, Queen Mary University of London, \\ Mile End Road, London, E1 4NS, UK. \\ ${ }^{b}$ The Lennard-Jones Laboratories, School of Chemical and Physical Science, Keele University, ST5 5BG, UK. \\ Email: m.watkinson@keele.ac.uk.
}

\begin{abstract}
The Golgi apparatus requires zinc for its normal function, but the role it plays in these processes at the sub-cellular level is not well-understood due to the lack of appropriate tools to image it. Herein, a small molecule Golgi apparatus targeted probe was developed to image mobile $\mathrm{Zn}^{2+}$. A trityl group was used to protect a Golgi apparatus targeting cysteine residue to increase membrane permeability, which was then removed in cellulo within 24 hours, revealing the free cysteine targeting motif to anchor the probe to the Golgi apparatus. The probe shows good photophysical properties, good selectivity and $\mathrm{Zn}^{2+}$ response over a wide range of $\mathrm{pH}$ as well as low cellular toxicity. The probe was shown to be capable of targeting the Golgi apparatus and responding to $\mathrm{Zn}^{2+}$ in a number of different cell lines and was also applied to monitor the change of concentration of mobile $\mathrm{Zn}^{2+}$ in the Golgi apparatus in response to oxidative stress.
\end{abstract}

\section{Introduction}

Zinc, as the second most abundant d-block metal in the human body, is involved in a wide range of biological processes. Whilst the majority of zinc is found in bound forms, there exists a pool of 'mobile' or 'free' zinc whose homeostasis is affected as a result of many diseases, including Alzheimer's disease $(A D),{ }^{1}$ prostate cancer, ${ }^{2}$ type 2 diabetes, ${ }^{3}$ and immune dysfunction and infection. ${ }^{4}$ To understand better the mechanisms of these pathological processes, it is desirable to be able to monitor $\mathrm{Zn}^{2+}$ changes at the cellular, or preferably, subcellular level. At the sub-cellular level, endoplasmic reticulum (ER), ${ }^{5,6}$ mitochondrial ${ }^{7,8}$ and lysosomal ${ }^{9,10}$ mobile $\mathrm{Zn}^{2+}$ have been widely studied using small molecule fluorescent probes, however, reports of its role in the Golgi apparatus are very rare. ${ }^{11}$ The Golgi apparatus works as a central station in cells, receiving secretory cargoes exported from the endoplasmic reticulum (ER), packing proteins into membrane-bound vesicles and sending them to their intra- and extra-cellular destinations. $\mathrm{Zn}^{2+}$ is integral to these processes for a variety of proteins, functioning in catalytic, regulatory, and structural roles. For example, it was found that zinc takes part in the interaction between the two main Golgi proteins GRASP55 and Golgin45, maintaining the normal morphology of the Golgi apparatus, ${ }^{12} \mathrm{Zn}^{2+}$ also coordinates with insulin monomers in the trans-Golgi network to package it into secretory granules, which are then released from pancreatic $\beta$-cells. ${ }^{13}$ Therefore, the breakdown of $\mathrm{Zn}^{2+}$ homeostasis in the Golgi apparatus is likely to be associated with a range of human disorders and the development of effective methods for its monitoring are required. 
Small molecule fluorescent probes are increasingly used in cellular analysis for a wide range of analytes and have many advantages as chemical biology tools, including their high sensitivity, stability and photophysical properties. ${ }^{14-18}$ However, their application in monitoring biological processes can be limited due to the failure to control their sub-cellular localisation. In the last two decades, there have been great efforts to increase their application through the development of a number of sub-cellular targeting probes. ${ }^{19-22}$ Among the many types of probe reported, the most effective and reliable strategy has been to incorporate specific targeting vectors into small molecular probes. Ceramide has been widely used for Golgi apparatus targeting and is employed in commercial stains for the Golgi apparatus such as NBD-ceramide and Bodipy-ceramide. However, it requires multi-step synthesis and also co-localises to the plasma membrane, which limits its application somewhat. Recently cysteine has been reported as a Golgi apparatus targeting unit, ${ }^{23,24}$ since galactosyltransferase and protein kinase $D$ were found to anchor to the Golgi region via their cysteine residues or cysteine rich domains. ${ }^{25,26}$ However, cysteine as a targeting vector has its limitations in small molecule probes since it is rather hydrophilic, ${ }^{27}$ and consequently not membrane permeable in some cases. Such poor membrane permeability has been classically addressed through the use of an ethyl ester protected carboxylic acid in the commercial zinc probe Zinquin, which was assumed to undergo esterase catalysed cleavage in cellulo, ${ }^{28}$ and this strategy has also been utilized to load cells with some other small molecule zinc probes. ${ }^{8}$

In this work, we present our recent efforts to develop a Golgi apparatus targeting $\mathrm{Zn}^{2+}$ probe, which can be used to study changes to mobile $\mathrm{Zn}^{2+}$ levels during oxidative stress. To overcome the poor membrane permeability of cysteine previously noted, ${ }^{27}$ a trityl protecting group was used to increase the hydrophobicity of the probe. Following passive diffusion into cells, the labile trityl group was removed intracellularly releasing the free cysteine, which then anchors the probe to the Golgi apparatus (Fig. 1).

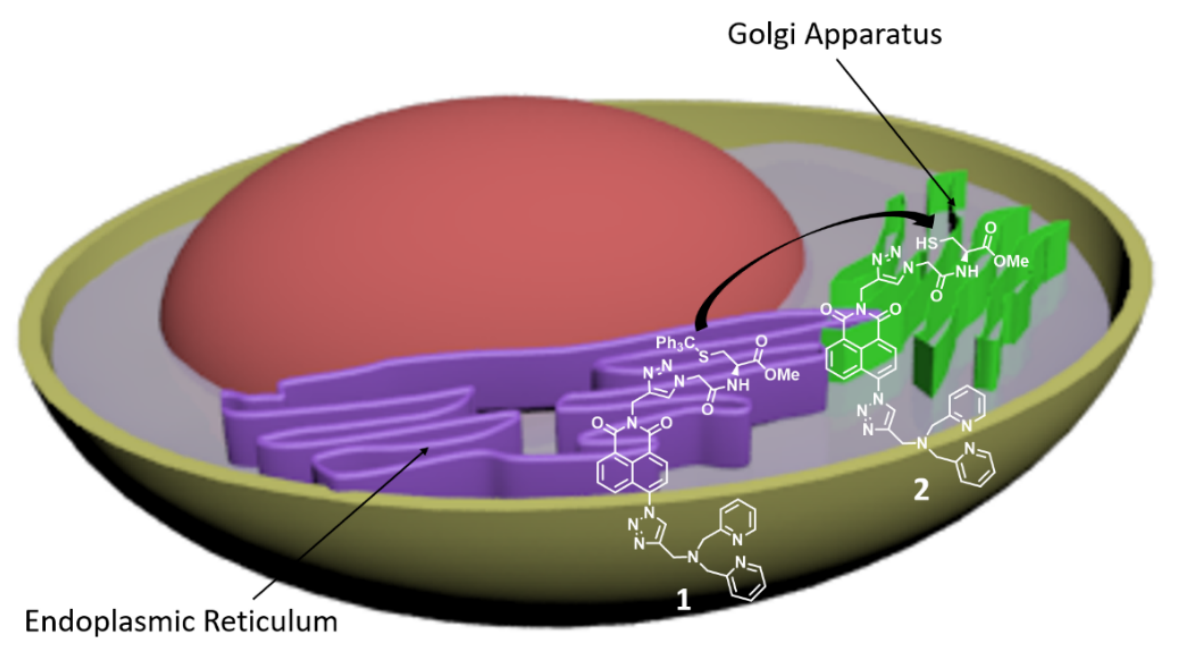

Fig. 1 The structure of the trityl protected $\mathrm{Zn}^{2+}$ probe, 1, and subsequent targeting of $\mathbf{2}$ to the Golgi apparatus after deprotection 


\section{Results and Discussion}

\section{Synthesis and characterization}

The synthetic route to both trityl protected probe $\mathbf{1}$ and its deprotected analogue $\mathbf{2}$ is shown in Scheme S1 (see Electronic Supporting Information (ESI)). Firstly, the cysteine thiol group was protected with a trityl group, as reported, ${ }^{29}$ to give S2. Following protection of the carboxylic acid as its methyl ester to give $\mathbf{S 3},{ }^{30}$ a 'click' azide group was then introduced to give amide S4. The trityl protected probe $\mathbf{1}$ was then readily obtained by stepwise double 'click' reactions following our originally reported procedures. ${ }^{31}$ Although the deprotected probe $\mathbf{2}$ was ultimately ineffective in cellulo, as expected, it was also isolated for further characterization and testing in vitro. All products were satisfactorily characterized by ${ }^{1} \mathrm{H},{ }^{13} \mathrm{C}$ NMR and IR spectroscopies, as well as high-resolution mass spectrometry.

\section{Photophysical studies}

Fluorescence $\mathrm{Zn}^{2+}$ titration of probe 1 (Fig. S2, ESI) shows it has a modest switch on response to $\mathrm{Zn}^{2+}$ with a dissociation constant of $3.28 \pm 0.12 \mathrm{nM}$ (Fig. S3 and Table S1, ESI), which is consistent with previous values we have observed in related probes with this zinc-binding motif and fluorophore. ${ }^{6,22} \mathrm{~A}$ titration of probe 1 with different equivalents of $\mathrm{Zn}^{2+}$ by ${ }^{1} \mathrm{H}$ NMR spectroscopy (Fig. S4-S6, ESI) was performed to further demonstrate the $\mathrm{Zn}^{2+}$ association. As previously reported, ${ }^{22}$ the ligand $\mathrm{N}, \mathrm{N}$-di-(2-picolyl)ethylenediamine (DPEN) and the bottom triazole (protons $\mathrm{H}_{\mathrm{a}-\mathrm{g}}$ ) are clearly involved in $\mathrm{Zn}^{2+}$ binding. In addition, a number of other proton signals (protons $\mathrm{H}_{\mathrm{h}-\mathrm{l}}$ and $\mathrm{H}_{\mathrm{m}-\mathrm{t}}$ ) display changes to their chemical shifts with increasing equivalents of $\mathrm{Zn}^{2+}$, however this is comparable to behaviour seen previously by us in related systems, ${ }^{22}$ which we attribute to global changes due to $\mathrm{Zn}^{2+}$ binding to DPEN. The deprotected probe 2 displays a similar switch on fluorescence response to $\mathrm{Zn}^{2+}$ (Fig. 2a) and its dissociation constant was similarly determined to be $3.30 \pm 0.13 \mathrm{nM}$ (Fig. S3 and Table S1, ESI), however, we were unable to perform a titration by ${ }^{1} \mathrm{H}$ NMR spectroscopy in this case due to poor solubility.

The fluorescence quantum yield of probe 1 was measured and was determined to be 0.0014 , while its complex with one equivalent of $\mathrm{Zn}^{2+}$ only increased to 0.0073 (Fig. 2b), which is much lower than that of the similar probes we have previously reported. ${ }^{6,22}$ This is likely to be due to the large amplitude vibrations of the trityl group, which increase non-radiative decay pathways. It is therefore unsurprising that when the trityl protecting group was removed, the quantum yield increased significantly and the quantum yield of probe 2 reached 0.046 and that of its complex with 1 equivalent of $\mathrm{Zn}^{2+}$ became 0.092 (Fig. 2b). This explanation was further supported by the fluorescence responses observed in solvent mixtures of increasing viscosity (Fig. S9) and temperature (Fig. S10). With increasing viscosity the fluorescence intensity of both probes increased significantly, however, the increase in intensity for probe $\mathbf{1}$ was exponential in nature, while it was more linear for probe 2. In contrast, when temperature was increased, the fluorescence intensity decreased, as expected, for both probes consistent with an increase in molecular vibrations although there were again differences observed between $\mathbf{1}$ and $\mathbf{2}$. Given that the quantum yield ratio between $\mathbf{2}$ and $\mathbf{1}$ is 33 we were optimistic that if $\mathbf{1}$ proved to be cell membrane permeable and could be 
converted to $\mathbf{2}$ in cellulo that this feature could prove to be experimentally useful since a significant increase in fluorescence output after deprotection of $\mathbf{1}$ and consequent Golgi apparatus localisation of $\mathbf{2}$ should result.

The fluorescence of probe $\mathbf{1}$ and $\mathbf{2}$ at different $\mathrm{pH}$ values was measured. Through non-linear curve fitting (Fig. S11-S12, ESI) of the fluorescence emission integration against $\mathrm{pH}$ with Equation S4, three $\mathrm{p} K_{\mathrm{a}}$ values of each probe were determined: for $1 \mathrm{p} K_{\mathrm{a} 1}=3.95 \pm 0.18, \mathrm{p} K_{\mathrm{a} 2}=$ $7.24 \pm 0.17$ and $\mathrm{p} K_{\mathrm{a} 3}=9.31 \pm 0.35$ whilst for 2 they were $\mathrm{p} K_{\mathrm{a} 1}=4.24 \pm 0.17, \mathrm{p} K_{\mathrm{a} 2}=6.13 \pm 0.18$ and $\mathrm{p} K_{\mathrm{a} 3}=8.39 \pm 0.34$. After addition of $\mathrm{Zn}^{2+}$, the fluorescence intensity increased in the $\mathrm{pH}$ range of 3.0-11.0 (Fig. S13, ESI), which more than covers the biologically relevant range and suggests that the probe should perform well in the cellular environment.

To specifically study the fluorescent stability of $\mathbf{1}$, time-dependent fluorescence measurements were undertaken at a range of cellularly relevant $\mathrm{pH} .{ }^{32}$ The probe was stirred in PBS buffer solution at a range of different $\mathrm{pH}$ values and its fluorescence response measured after 1, 6, 12 and 24 hours (Fig. S14, ESI). The fluorescence at pH 6.0 (the pH of the trans-Golgi) was higher than that at 7.2 (the $\mathrm{pH}$ of cytoplasm and endoplasmic reticulum) presumably due to the more acidic nature of the solutions, but all of them remained at the same level after 24 hours, indicating the probe to be stable at the relevant cellular $\mathrm{pH}$ values for prolonged periods. Furthermore, as no obvious fluorescence increase was observed with time under the acidic conditions it also suggests that there is no S-trityl deprotection in solution at these $\mathrm{pH}$ values, indicating that any deprotection that may occur in cellulo is likely to be metabolic, rather than protic, in nature.

The selectivity of the probe was investigated in the presence of a range of other cations. As shown in Fig. S15, the addition of a variety of cations has little or no effect on the fluorescence response of 1 , even $\mathrm{Cd}^{2+}$, which is a common problem for this kind of binding motif. ${ }^{5,7}$ After subsequent addition of $\mathrm{Zn}^{2+}$, the probe 1 was still quenched in the presence of $\mathrm{Co}^{2+}, \mathrm{Cu}^{2+}$ and $\mathrm{Ni}^{2+}$, but this is not concerning as these cations are not abundant in free forms in biological environments. Probe $\mathbf{2}$ (Fig. S16, ESI) shows broadly the similar selective properties to $\mathbf{1}$. For the majority of cations tested, particularly the physiologically relevant cations $\left(\mathrm{Na}^{+}, \mathrm{K}^{+}, \mathrm{Mg}^{2+}\right.$, $\left.\mathrm{Ca}^{2+}\right)$, both probes displayed nearly complete recovery after addition of $\mathrm{Zn}^{2+}$, meaning it has the potential to be applied in cellulo to image $\mathrm{Zn}^{2+}$.

a)

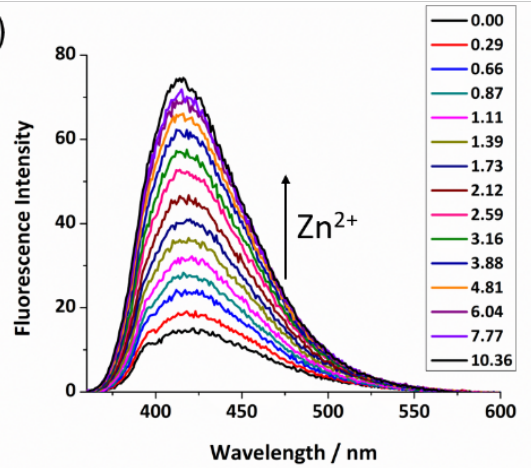

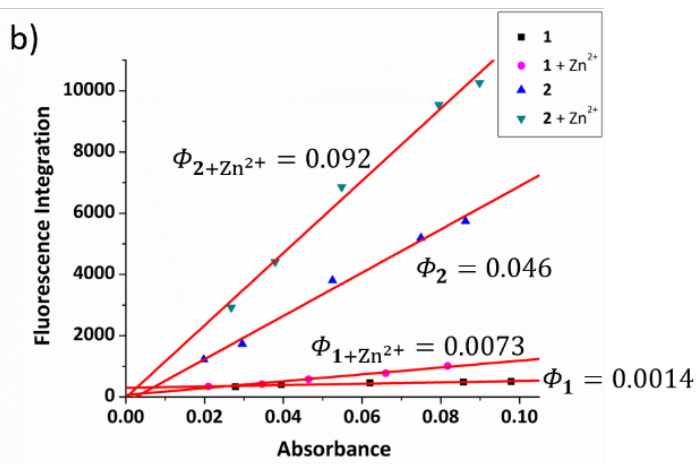

b)

Fig. 2 a) The fluorescence spectra of $2(1 \mu M)$ at different concentrations of free $\mathrm{Zn}^{2+}(\mathrm{nM}, 50 \mathrm{mM}$ HEPES buffer, pH 7.2, 0.1 M KCl); b) Linear plots for $\mathbf{1}, \mathbf{2}$ and their $\mathrm{Zn}^{2+}$ complexes to obtain quantum yield values ( $0.1 \mathrm{mM}$ HEPES buffer, $\mathrm{pH} 7.4, \lambda_{\mathrm{ex}}=346 \mathrm{~nm}, \lambda_{\mathrm{em}}=414 \mathrm{~nm}$, slit width: $5 / 5 \mathrm{~nm}$ ). 


\section{DFT calculations}

DFT calculations were carried out to study the association between probe and $\mathrm{Zn}^{2+}$ in silico (Fig. S17-S18, ESI). The calculated optimised structures supported the bottom triazole groups being involved in $\mathrm{Zn}^{2+}$ binding in addition to the DPEN ligands, as also observed by ${ }^{1} \mathrm{H} N M R$ titrations.

In order to establish the excitation and emission profiles of the probe, TDDFT simulations were undertaken. The calculated absorption energy from ground state $S_{0}$ to excited state $S_{1}$, and the emission energy from $S_{1}$ to $S_{0}$ were in agreement with the experimental data (Table S2-S3, ESI). The $\mathrm{S}_{1}-\mathrm{S}_{0}$ electron density transition map (Fig. S19-S20, ESI) shows the electron changes mainly occur at the naphthalimide moiety and the vicinal triazole. After binding with $\mathrm{Zn}^{2+}$, there is only a slight decrease of electron density on the triazole, and the oscillator strength is not significantly affected compared to the free probe. Therefore, the emission increase of the complex is related to a reduced decay through nonradiative pathways after binding with $\mathrm{Zn}^{2+}$. The stabilisation of the complex hinders large amplitude vibrations in the vicinity of the fluorophore, which hampers the access to nonradiative mechanisms and increases the quantum yield of fluorescence. The restriction of intramolecular rotations can also hinder the access to low energy conical intersections associated with ultrafast decay to the ground state. This theory was used to explain similar previous findings by us ${ }^{6,22,31}$ and has been discussed elsewhere. ${ }^{33,34}$

\section{Sub-cellular localisation}

As the probes showed promising photophysical properties in solution, their application in cells was also assessed. Firstly, the toxicity of $\mathbf{1}$ and $\mathbf{2}$ was measured through the alamarBlue cell viability assay, and after 24 hours' incubation with both probes, the viability of HeLa cells (Fig. S21-S22, ESI) did not show any obvious decrease even with probe concentrations up to $50 \mu \mathrm{M}$, indicating that they are not cytotoxic. However, initial fluorescence microscopy tests of the probes in HeLa cells (Fig. S23, ESI) showed that the cells incubated with $\mathbf{2}$ do not display any intracellular fluorescence, indicating that $\mathbf{2}$, as expected due to its hydrophilic nature, ${ }^{27}$ is not membrane permeable. In contrast, cells incubated with 1 clearly showed evidence of probe internalisation. Therefore, further cell experiments only focused on probe 1.

To test the Golgi apparatus targeting ability of 1, HeLa cells were incubated in its presence for 1 hour and were then co-incubated with different organelle tracker dyes for a certain time period (normally 15-30 minutes). This revealed that the fluorescence response of 1 was almost exclusively only observed in the endoplasmic reticulum (ER) rather than the Golgi apparatus (Fig. 3) and was of relatively low intensity. We speculated that this was because the trityl protecting group was not removed in this time in cellulo and that a longer incubation time was required to facilitate this and subsequent localisation into the Golgi apparatus. Indeed, this proved to be the case and when the incubation time was extended to 24 hours the fluorescent response showed a significant increase in intensity (Fig. S24), with excellent co-localisation with Bodipy TR ceramide observed, a commercial Golgi tracker red reagent, with no evidence of ER fluorescence as had been the case for the shorter incubation periods. Given the apparent metabolic nature of this process (vide infra), we speculated that $S$ - 
adenosyl-L-homocysteine (SAH) hydrolase ${ }^{35}$ or a similar enzyme in it the wider S-adenosyl-Lmethionine family ${ }^{36}$ was responsible for the removal of the $S$-trityl protecting group. Therefore, a further control experiment in which cells were treated with probe $\mathbf{1}$ for $\mathbf{2 4}$ hours in the presence of a well-known SAH hydrolase inhibitor, adenosine dialdehyde (Adox) ${ }^{37,38}$ was undertaken. This clearly showed no evidence of Golgi apparatus accumulation and fluorescence intensity increase (Fig. 3 and S24) and supports the integrity of the S-trityl protecting group in the presence of the inhibitor. No change in the response of 1 in the presence of the inhibitor was observed in control experiments in vitro (Fig. S25, ESI). The same result was also observed in the MCF-7 cell line (Fig. S26, ESI), suggesting this behaviour to be generic and consistent with the 'protect to detect' hypothesis.

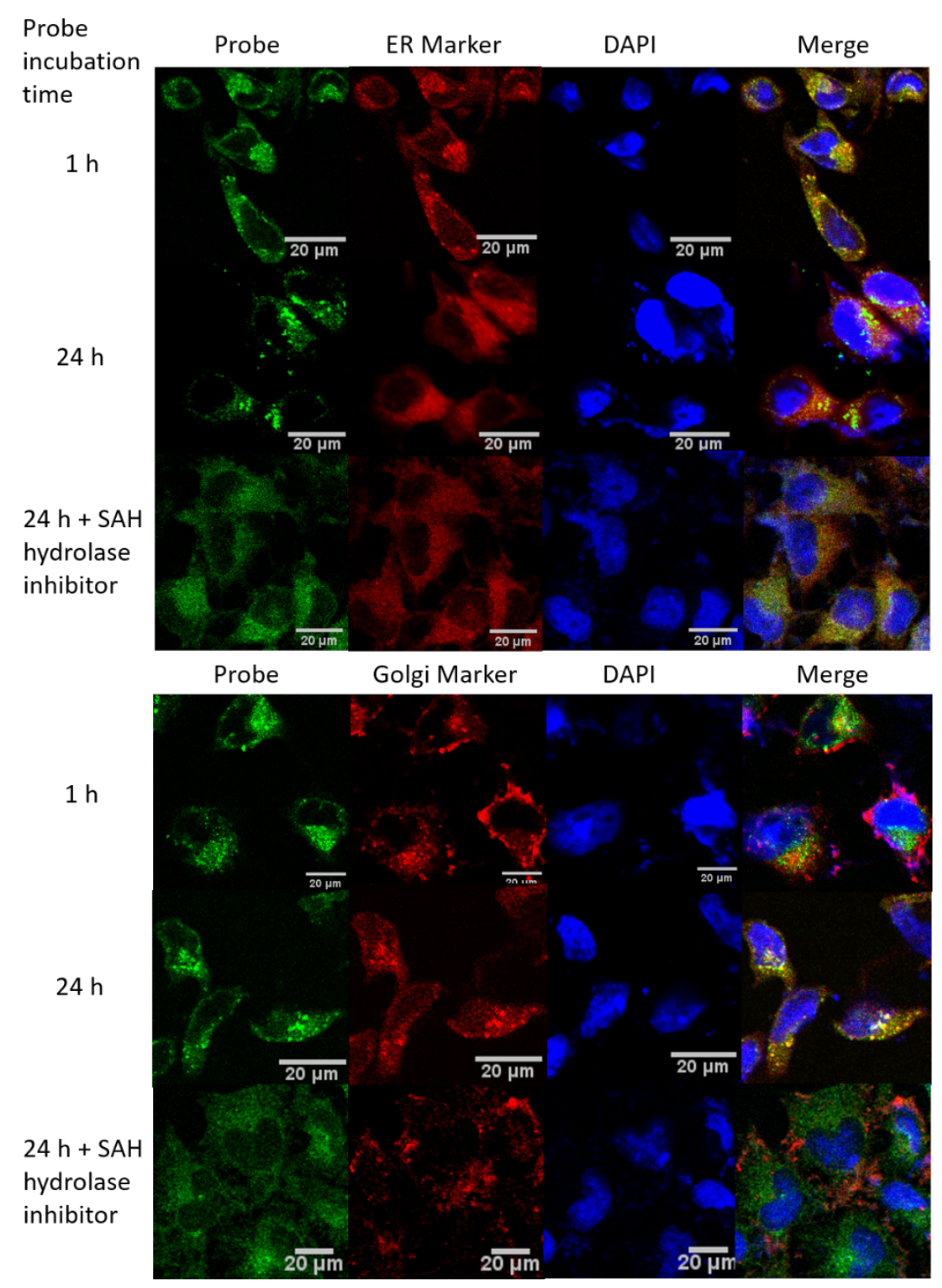

Fig. 3 Confocal microscopy images of HeLa cells after treatment with $1\left(10 \mu \mathrm{M}, \lambda_{\mathrm{ex}}=488 \mathrm{~nm}, \lambda_{\mathrm{em}}=\right.$ $509 \mathrm{~nm})$ for $1 \mathrm{~h}$ or $24 \mathrm{~h}$, and in presence of SAH hydrolase inhibitor adenosine dialdehyde $(30 \mu \mathrm{M})$ compared to ER marker or Golgi marker $\left(\lambda_{\mathrm{ex}}=594 \mathrm{~nm}, \lambda_{\mathrm{em}}=620 \mathrm{~nm}\right)$. (Scale bars $=20 \mu \mathrm{m}$ ) 
The Pearson's correlation coefficient was measured by comparing probe 1 (GFP channel) after 24 hours of incubation to different organelle trackers (RFP channel). In HeLa cells (Fig. 4), the fluorescence of 1 showed excellent correlation with that of Bodipy TR ceramide, giving a Pearson's coefficient of 0.92 . The values of negative control groups were much lower, with values of $0.42,0.38$ and 0.59 when compared to commercial ER, Mito and Lyso tracker red dyes respectively. Similar results were obtained in the breast cancer cell line MCF-7 (Fig. S27, ESI) and a sweat gland tissue cell line EC23 (Fig. S28, ESI), revealing the probe's general Golgitargeting capability

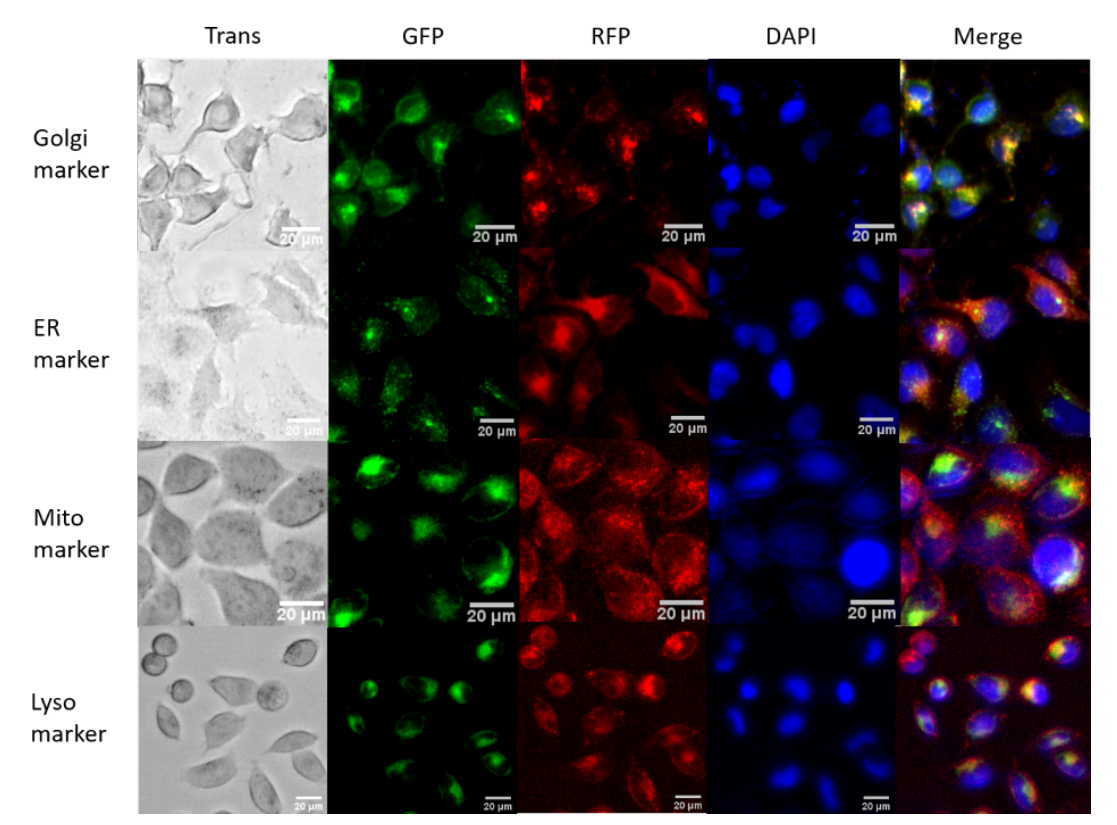

Fig. 4 The colocalisation images of HeLa cells incubated with probe $1\left(10 \mu \mathrm{M}\right.$, GFP filter: $\lambda_{\mathrm{ex}}=$ $470 / 30 \mathrm{~nm}, \lambda_{\mathrm{em}}=530 / 50 \mathrm{~nm}$ ) and organelle tracker red dyes (RFP filter: $\lambda_{\mathrm{ex}}=530 / 40 \mathrm{~nm}, \lambda_{\mathrm{em}}=$ $605 / 55 \mathrm{~nm}$ ). (Scale bars $=20 \mu \mathrm{m}$ )

\section{$\mathrm{Zn}^{2+}$ response in cells}

The response of probe 1 in cells was recorded to demonstrate its ability to respond to variation in $\mathrm{Zn}^{2+}$ levels in the Golgi apparatus. As shown in Fig. 5, the fluorescence of $\mathbf{1}$ in HeLa cells increased significantly after the addition of zinc pyrithione, a membrane permeable zinc complex, indicating it has switch on fluorescence response to $\mathrm{Zn}^{2+}$ in cellulo. When $N, N, N^{\prime}, N^{\prime}-$ tetrakis(2-pyridinylmethyl)-1,2-ethanediamine (TPEN), a well-known chelator of 'free' zinc, ${ }^{31}$ was added, the fluorescence was almost completely quenched. Similar results were also observed in MCF-7 (Fig. S29, ESI) and EC23 cell lines (Fig. S30, ESI), and the fluorescence intensity read from the images is shown in Fig. S31 in the ESI. 


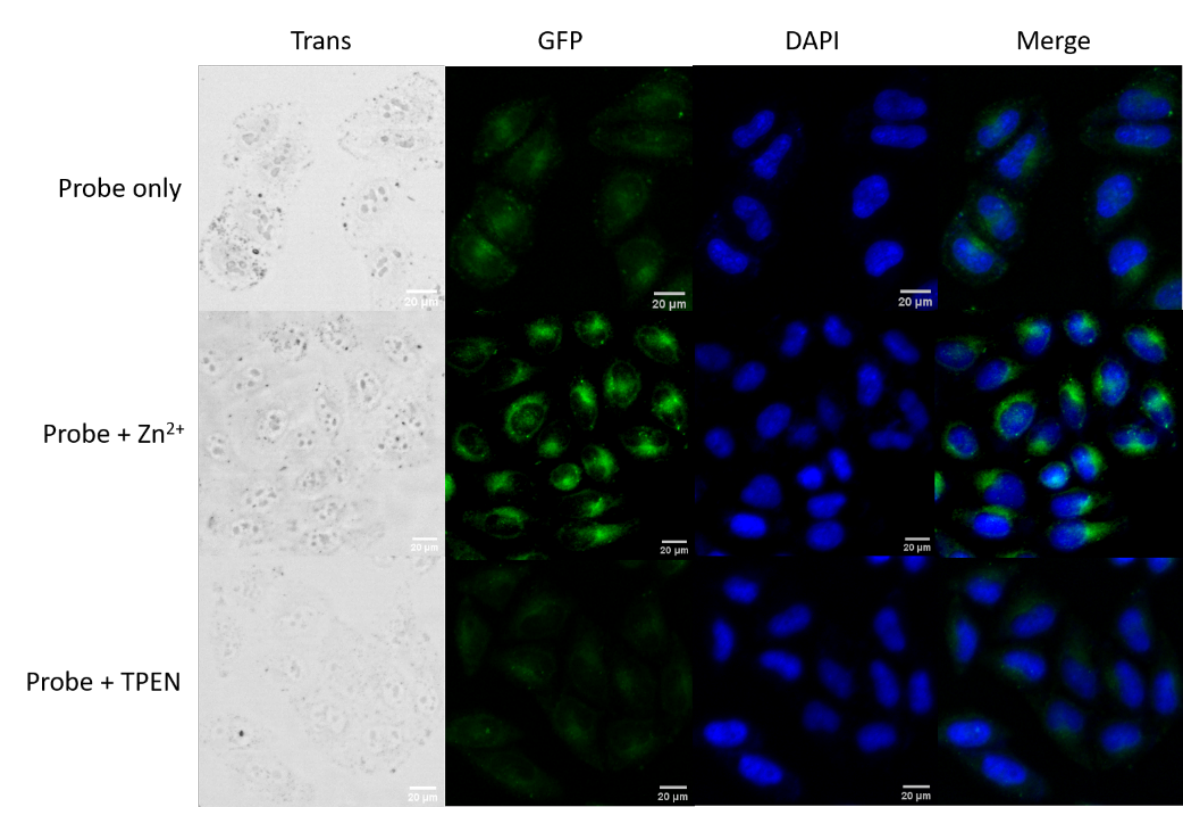

Fig. 5 Fluorescence microscopy images of HeLa cells treated with $1(10 \mu \mathrm{M}), 1(10 \mu \mathrm{M})$ with zinc pyrithione $(50 \mu \mathrm{M})$, and $1(10 \mu \mathrm{M})$ with TPEN $(50 \mu \mathrm{M})$. (Scale bars $=20 \mu \mathrm{m}$, GFP filter: $\lambda_{\mathrm{ex}}=470 / 30 \mathrm{~nm}$, $\left.\lambda_{\mathrm{em}}=530 / 50 \mathrm{~nm}\right)$

\section{$\mathrm{Zn}^{2+}$ level changes during oxidative stress}

Given that 1 is able to image $\mathrm{Zn}^{2+}$ in the Golgi apparatus in three cell lines and respond to variations in its levels induced by the addition of zinc pyrithione or TPEN, we sought to explore its application to monitor mobile $\mathrm{Zn}^{2+}$ changes in the Golgi apparatus during changes to redox status, as this has not been investigated widely due to the lack of an effective tool to do so. MCF-7 cells, after 24 hours' incubation with 1 , were first treated with $\mathrm{H}_{2} \mathrm{O}_{2}$ to induce oxidative stress and the intensity of the fluorescence response in the cells monitored against time (Fig. 6a). The fluorescence response was observed to be significantly reduced after the addition of $\mathrm{H}_{2} \mathrm{O}_{2}$ with levels stabilising after approximately 30 mins. Then glutamine was added to reverse oxidative status, and the fluorescence intensity then remained stable. In contrast, when glutamine was first introduced and $\mathrm{H}_{2} \mathrm{O}_{2}$ subsequently added the fluorescence remained largely stable with only a small decrease of its intensity (Fig. 6b). The reasons for the reduced fluorescence response observed in Fig. 6a may, in part, be due to the known antioxidant role zinc plays since it is a cofactor of the superoxide dismutase enzyme, which regulates the detoxification of reactive oxygen species, protecting cells against the oxidative stress. ${ }^{3}$ However, it has also been reported that the concentration of mobile $\mathrm{Zn}^{2+}$ increases in the mitochondria ${ }^{39}$ and lysosome ${ }^{40}$ under oxidative stress induced by $\mathrm{H}_{2} \mathrm{O}_{2}$. Metallothionein, which is mainly localised to the membrane of the Golgi apparatus, ${ }^{41}$ is known to play an important role in the regulation of $\mathrm{Zn}^{2+}$ homeostasis, as well as providing protection against oxidative stress in cells. ${ }^{42}$ We therefore assume that under oxidative stress, metallothionein transports $\mathrm{Zn}^{2+}$ from the Golgi apparatus to the mitochondria and lysosome to function. In contrast, if glutamine was first added, the addition of $\mathrm{H}_{2} \mathrm{O}_{2}$ should not cause high levels of oxidative stress, therefore zinc levels would not be reduced and therefore no significant 
fluorescence decrease would be expected, as observed. Similar results were also observed in HeLa cells, (Fig. S32, ESI), indicating this process is likely to be general among different cell lines. Finally, an in vitro control experiment was undertaken to exclude the effect of $\mathrm{H}_{2} \mathrm{O}_{2}$ and glutamine themselves on the fluorescence response of the both $\mathbf{1}$ and $\mathbf{2}$, which showed there was no difference in response when the two reagents were added separately in the presence of zinc (Fig. S33 and Fig. S34, ESI). Therefore, it appears reasonable to propose that there is a lowering of the concentration of mobile $\mathrm{Zn}^{2+}$ in the Golgi apparatus in response to oxidative stress.

a)

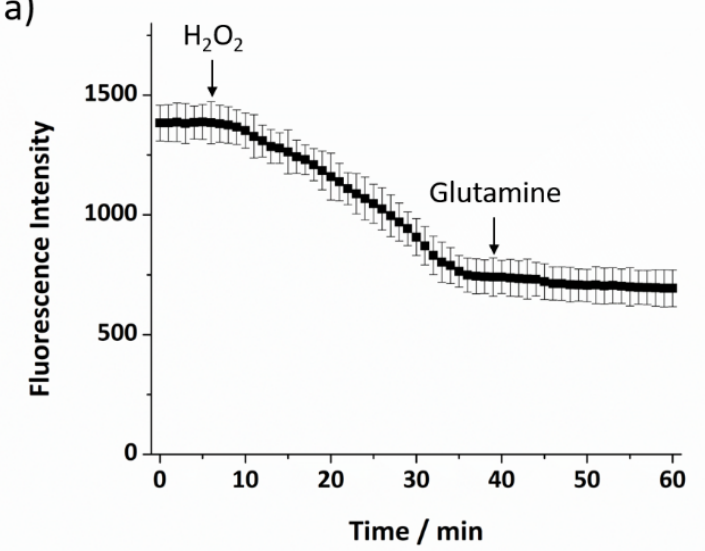

b)

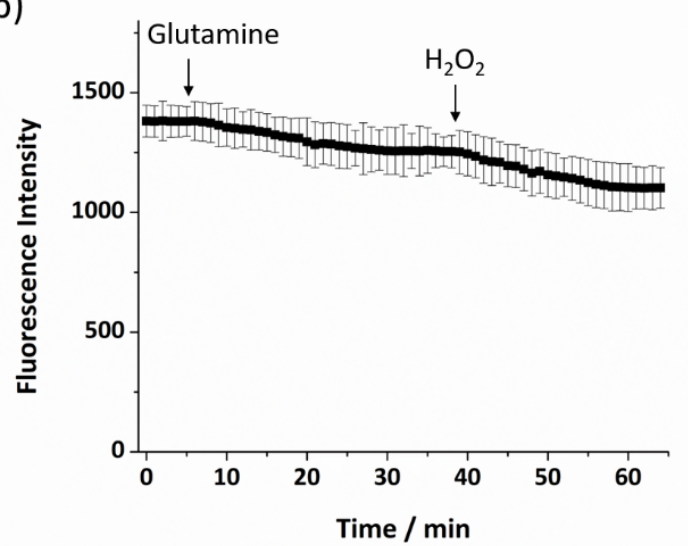

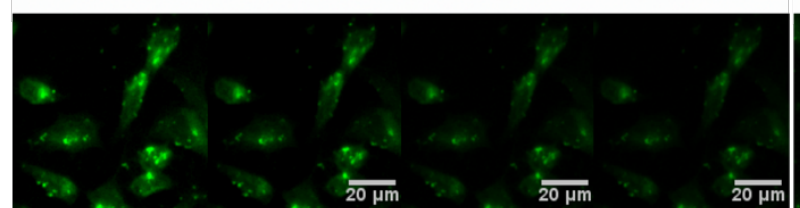

$0 \min$

$20 \mathrm{~min}$

$40 \mathrm{~min}$

$60 \mathrm{~min}$

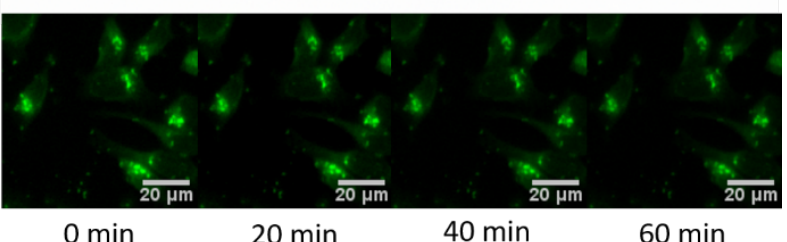

Fig. 6 Fluorescence response in MCF-7 cells treated with $1(10 \mu \mathrm{M})$ for 24 hours and then with a) $\mathrm{H}_{2} \mathrm{O}_{2}$ $(200 \mu \mathrm{M})$ and then glutamine $(200 \mu \mathrm{M})$ or b) glutamine $(200 \mu \mathrm{M})$ and then $\mathrm{H}_{2} \mathrm{O}_{2}(200 \mu \mathrm{M})$ with images taken at different times. (Scale bars $=20 \mu \mathrm{m}$, GFP filter: $\lambda_{\mathrm{ex}}=470 / 30 \mathrm{~nm}, \lambda_{\mathrm{em}}=530 / 50 \mathrm{~nm}$ )

\section{Conclusion}

In summary, a Golgi apparatus targeted $\mathrm{Zn}^{2+}$ probe 1 was successfully synthesized that is able to undergo passive membrane diffusion as a result of the lipophilic nature of the $S$-trityl protecting group. It is apparent that initial probe localisation in cellulo occurs in the ER, however, after 24 hours the fluorescence response increases significantly, presumably due to $S$-trityl group deprotection forming 2, which displays a quantum yield that is 33-fold higher than $\mathbf{1}$, and the probe consequently becomes anchored to the Golgi apparatus. The probe shows excellent specificity for targeting of the Golgi apparatus and the ability to image mobile $\mathrm{Zn}^{2+}$ in different cell lines. This allowed it to be applied in the monitoring of changes to mobile $\mathrm{Zn}^{2+}$ levels in the Golgi apparatus in response to oxidative stress induced by $\mathrm{H}_{2} \mathrm{O}_{2}$, which indicated that the concentration of mobile $\mathrm{Zn}^{2+}$ decreases. This suggests that the probe may provide an effective tool to probe changes to mobile $\mathrm{Zn}^{2+}$ that occur in the Golgi apparatus in response to cellular stress in a number of diseases states.

\section{Conflicts of interest}


There are no conflicts to declare.

\section{Acknowledgements}

DFT calculations were performed on the QMUL Apocrita facility. LF is grateful to the Chinese Scholarship Council for the provision of a PhD studentship. We would like to thank Dr. Giuseppe Trigiante and Dr. Hemanth Tummala for their help and advice with cell experiments and Prof. Mike Philpott for access to his laboratory to undertake them.

\section{References}

1 S. L. Sensi, A. Granzotto, M. Siotto and R. Squitti, Trends Pharmacol. Sci., 2018, 39, 1049-1063.

2 L. C. Costello and R. B. Franklin, Arch. Biochem. Biophys., 2016, 611, 100-112.

3 P. Chabosseau and G. A. Rutter, Arch. Biochem. Biophys., 2016, 611, 79-85.

4 A. S. Prasad, Curr. Opin. Clin. Nutr. Metab. Care, 2009, 12, 646-652.

5 W. Lin, D. Buccella and S. J. Lippard, J. Am. Chem. Soc., 2013, 135, 13512-13520.

6 L. Fang, G. Trigiante, R. Crespo-Otero, C. S. Hawes, M. P. Philpott, C. R. Jones and M. Watkinson, Chem. Sci., 2019, 10, 10881-10887.

7 H. M. Kim, C. S. Lim, G. Masanta, H. J. Kim, B. R. Cho and J. H. Han, J. Am. Chem. Soc., 2011, 133, 5698-5700.

8 W. Chyan, D. Y. Zhang, S. J. Lippard and R. J. Radford, Proc. Natl. Acad. Sci., 2014, 111, 143-148.

9 Q. Liu, D. Zhu, H. Jiang, L. Xue and G. Li, Inorg. Chem., 2012, 51, 10842-10849.

10 C. Du, S. Fu, X. Wang, A. C. Sedgwick, W. Zhen, M. Li, X. Li, J. Zhou, Z. Wang, H. Wang and J. L. Sessler, Chem. Sci., 2019, 10, 5699-5704.

11 H. Singh, H. W. Lee, C. H. Heo, J. W. Byun, A. R. Sarkar and H. M. Kim, Chem. Commun., 2015, 51, 12099-12102.

12 H. Wu and J. Zhao, Metallomics, 2019, 11, 1984-1987.

13 Y. V. Li, Endocrine, 2014, 45, 178-189.

14 M. H. Lee, J. S. Kim and J. L. Sessler, Chem. Soc. Rev., 2015, 44, 4185-4191.

15 H. M. Kim and B. R. Cho, Chem. Rev., 2015, 115, 5014-5055.

16 H. W. Liu, L. Chen, C. Xu, Z. Li, H. Zhang, X. B. Zhang and W. Tan, Chem. Soc. Rev., 2018, 47, 71407180.

17 J. Chan, S. C. Dodani and C. J. Chang, Nat. Chem., 2012, 4, 973-984.

18 L. Wang, M. S. Frei, A. Salim and K. Johnsson, J. Am. Chem. Soc., 2019, 141, 2770-2781.

19 H. Zhu, J. Fan, J. Du and X. Peng, Acc. Chem. Res., 2016, 49, 2115-2126.

20 W. Xu, Z. Zeng, J. H. Jiang, Y. T. Chang and L. Yuan, Angew. Chem. Int. Ed., 2016, 55, 13658-13699.

21 J. Pancholi, D. J. Hodson, K. Jobe, G. A. Rutter, S. M. Goldup and M. Watkinson, Chem. Sci., 2014, 5, 3528-3535. 
22 L. Fang, G. Trigiante, R. Crespo-Otero, M. P. Philpott, C. R. Jones and M. Watkinson, Org. Biomol. Chem., 2019, 17, 10013-10019.

23 R. S. Li, P. F. Gao, H. Z. Zhang, L. L. Zheng, C. M. Li, J. Wang, Y. F. Li, F. Liu, N. Li and C. Z. Huang, Chem. Sci., 2017, 8, 6829-6835.

24 W. Zhang, J. Zhang, P. Li, J. Liu, D. Su and B. Tang, Chem. Sci., 2019, 10, 879-883.

25 D. Aoki, N. Lee, N. Yamaguchi, C. Dubois and M. N. Fukuda, Proc. Natl. Acad. Sci. U. S. A., 1992, 89, 4319-4323.

26 Y. Maeda, G. V. Beznoussenko, J. Van Lint, A. A. Mironov and V. Malhotra, EMBO J., 2001, 20, 59825990.

27 H. Wang, Z. He, Y. Yang, J. Zhang, W. Zhang, W. Zhang, P. Li and B. Tang, Chem. Sci., 2019, 10, 10876-10880.

28 P. Coyle, P. D. Zaleewski, J. C. Philcox, I. J. Forbes, A. D. Ward, S. F. Lincoln, I. Mahadevan and A. M. Rofe, Biochem. J., 1994, 303, 781-786.

29 J. Nanda, B. Adhikari, S. Basak and A. Banerjee, J. Phys. Chem. B, 2012, 116, 12235-12244.

30 T. M. Vishwanatha, K. Kurpiewska, J. Kalinowska-Tluścik and A. Dömling, J. Org. Chem., 2017, 82, 9585-9594.

31 L. Fang, G. Trigiante, C. J. Kousseff, R. Crespo-Otero, M. P. Philpott and M. Watkinson, Chem. Commun., 2018, 54, 9619-9622.

32 A. Asokan and M. J. Cho, J. Pharm. Sci., 2002, 91, 903-913.

33 D. Escudero, Acc. Chem. Res., 2016, 49, 1816-1824.

34 Y. Tu, J. Liu, H. Zhang, Q. Peng, J. W. Y. Lam and B. Z. Tang, Angew. Chem. Int. Ed., 2019, 131, 15053-15056.

35 Y. Takata, T. Yamada, Y. Huang, J. Komoto, T. Gomi, H. Ogawa, M. Fujioka and F. Takusagawa, J. Biol. Chem., 2002, 277, 22670-22676.

36 J. T. Jarrett, J. Biol. Chem., 2015, 290, 3972-3979.

37 A. Deussen, H. G. E. Lloyd and J. Schrader, J. Mol. Cell. Cardiol., 1989, 21, 773-782.

38 J. H. Kim, J. H. Kim, S. C. Kim, Y. S. Yi, W. S. Yang, Y. Yang, H. G. Kim, J. Y. Lee, K. H. Kim, B. C. Yoo, S. Hong and J. Y. Cho, Biochem. Pharmacol., 2013, 86, 1285-1300.

39 Z. Liu, C. Zhang, Y. Chen, W. He and Z. Guo, Chem. Commun., 2012, 48, 8365-8367.

40 H. Zhu, J. Fan, S. Zhang, J. Cao, K. Song, D. Ge, H. Dong, J. Wang and X. Peng, Biomater. Sci., 2014, 2, 89-97.

41 L. Ercolani, J. L. Stow, J. F. Boyle, E. J. Holtzman, H. Lin, J. R. Grove and D. A. Ausiello, Proc. Natl. Acad. Sci. U. S. A., 1990, 87, 4635-4639.

42 B. Ruttkay-Nedecky, L. Nejdl, J. Gumulec, O. Zitka, M. Masarik, T. Eckschlager, M. Stiborova, V. Adam and R. Kizek, Int. J. Mol. Sci., 2013, 14, 6044-6066. 